\title{
Detection of Metallo- $\beta$-Lactamases (MBLs) Producing Pseudomonas aeruginosa Isolates in Tehran Hospitals, Iran
}

\author{
Mohsen Tabasi ${ }^{1,2}$, Reza Azizian³, Mohammad Reza Eskandarion², Mehri Habibi ${ }^{1}$, Mohammad Reza Asadi \\ Karam $^{1 *}$
}

${ }^{1}$ Department of Molecular Biology, Pasteur Institute of Iran, Tehran, Iran; ${ }^{2}$ Iranian Legal Medicine Research Center, Legal Medicine Organization, Tehran, Iran; ${ }^{3}$ Massoud Clinical Laboratory, Tehran, Iran

Received Mar 05, 2018; Accepted Mar 14, 2018

\begin{abstract}
Introduction: The strains of Pseudomonas aeruginosa are known as an opportunistic pathogen that can cause infections in humans and animals. Metallo- $\beta$-lactamases (MBLs) are the most significant factors of resistance to carbapenem antibiotics in these bacteria. This study was designed to identify the MBLs producing $P$. aermginosa isolates in three hospitals of Tehran, Iran. Methods: Totally, we obtained 665 samples from patients hospitalized in three hospitals in Tehran, Iran. Antibioticsusceptibility test of the $P$. aeruginosa isolates was done based on Kirby-Bauer disk diffusion test. The Minimum Inhibitory Concentration (MIC) of the isolates was performed using agar dilution method, and IPM-EDTA test identified MBL producing isolates. Results: Among the examined isolates, 473 (71.1\%) were P. aeruginosa. Among these, 306 (64.7\%) were resistant to imipenem, and $289(94.5 \%)$ were MBL producers. Furthermore, the resistance rate of the isolates to other antibiotics was amikacin $(26 \%)$, tobramycin $(24.95 \%)$, ceftazidime $(23.05 \%)$, gentamicin $(22.83 \%)$, carbenicillin $(21.14 \%)$, and ceftizoxime (18.19\%). The MICs of imipenem and ceftazidime for the majority of the isolates were $4 \mu \mathrm{g} / \mathrm{ml}$ and $>128 \mu \mathrm{g} / \mathrm{ml}$, respectively. Conclusion: This study confirmed previous reports on the increased rate of MBL-mediated resistance in $P$. aeruginosa isolates worldwide. Therefore, detection of resistance patterns for these isolates, particularly MBLs, is necessary for prevention and control of Pseudomonas associated infections. J Med Microbiol Infect Dis, 2017, 5 (3-4): 47-50. DOI: 10.29252/JoMMID.5.3.4.47
\end{abstract}

Keywords: Psendomonas aeruginosa, Metallo- $\beta$-lactamase, IPM-EDTA, MIC.

\section{INTRODUCTION}

Nosocomial infections are among the most critical issues in developed and developing countries worldwide [1]. Pseudomonas aeruginosa is usually the third significant cause of hospital infections after Staphylococcus aureus and Escherichia coli [2]. For example, a study in Netherland indicated that $P$. aeruginosa strains were the causative agents of $27-37 \%$ of wound infections [3]. The spread of antibiotic resistance especially in healthcare centers has made control of pathogens difficult. Nowadays, the emergence of antibiotic-resistant strains has become a significant challenge in patients' treatment. The isolation rate of Multi-Drug Resistant (MDR) P. aeruginosa strains from the hospital environments, and the personnel hands are increasing in some countries [4]. For example, at one burn center in Tehran, Iran, the frequency of $P$. aeruginosa among the patients was $73.9 \%$, and more than $95 \%$ of the isolates showed resistance to gentamicin, carbenicillin, trimethoprim/sulfamethoxazole, ceftizoxime and tetracycline [5].

Carbapenems such as imipenem and meropenem are among the essential antimicrobial agents used for the treatment of human infections especially those caused by MDR-P. aeruginosa in hospital settings [6, 7]. Recently, resistance to carbapenems particularly in clinical isolates was reported to be related to the reduction of drug penetration or production of carbapenems hydrolyzing enzymes like Metallo- $\beta$-lactamases (MBLs) [8]. The first report on MBLs was from Japan, followed by reports from different countries in Asia, Europe, Australia and America $[9,10]$. These enzymes have a broad substrates spectrum and can hydrolyze all beta-lactam compounds except monobactams (aztreonam). These enzymes are transmitted by integrons and can merge in plasmids or chromosomes, and hence are transferred to different bacteria such as Enterobacteriaceae family and Pseudomonas strains [11-13].

Some reports linked the rise of carbapenem-resistant $P$. aeruginosa isolates to MBLs production as the MBL producing $P$. aeruginosa strains are reported worldwide [14]. MBL producing $P$. aeruginosa strains are considered as a significant threat in healthcare centers, due to the ability of gene transfer and long-term colonization in hospitals. Thus, rapid detection of these strains and reporting them in hospital settings can lead to better control measures in order to prevent their spread and also choose effective antibiotics.

*Correspondence: Mohammad Reza Asadi Karam

Department of Molecular Biology, Pasteur Institute of Iran, No. 69, Pasteur Ave, Tehran, Iran, 1316943551.

Email: m_asadi12@yahoo.com

Tel: +98 (21) $66953311 \quad$ Fax: +98 (21) 66492619 
Therefore, the primary purpose of this investigation was to determine and evaluate the production of MBLs among $P$. aeruginosa isolates obtained from several hospitals in Tehran, Iran.

\section{MATERIAL AND METHODS}

Collection and isolation of $P$. aeruginosa. From March 2015 to February 2016, 665 clinical samples including urine, sputum, wound, blood, and CSF were collected from the hospitalized patients in three hospitals in Tehran, Iran. Informed consent was obtained from all human adult participants and the parents or legal guardians of minors. For identification of $P$. aeruginosa isolates, the samples were cultured in blood agar and EMB medium (Merck, Germany) and then identified by biochemical standard tests such as growth in citrate, reaction in TSI, Oxidization Fermentation (OF) test and pyocyanin pigment production on Muller Hinton agar (MHA) (Merck, Germany) and growth in $42^{\circ} \mathrm{C}$. The confirmed isolates were stored at $70^{\circ} \mathrm{C}$ in a liquid medium of Luria-Bertani (LB) (Merck, Germany) with $20 \%$ glycerol for subsequent steps.

Antibiotic susceptibility patterns. Antibiotic resistance patterns of the $P$. aeruginosa isolates were determined by disk diffusion method based on Clinical and Laboratory Standards Institute (CLSI) recommendation (2008) [15] using the antibiotics, imipenem (10 $\mu \mathrm{g})$, meropenem $(10 \mu \mathrm{g})$, ceftazidime $(30 \mu \mathrm{g})$, carbenicillin $(100$ $\mu \mathrm{g})$, tobramycin $(10 \mu \mathrm{g})$, amikacin $(30 \mu \mathrm{g})$ ceftizoxime (30 $\mu \mathrm{g})$, and gentamicin $(10 \mu \mathrm{g})$ (Mast Co., UK). Inhibition zone diameter $(\mathrm{mm})$ of each antibiotic disc was measured, and the isolates were classified as resistant, intermediate or susceptible. Pseudomonas aeruginosa ATCC 27853 was used as a control strain.

Determination of Minimum Inhibitory Concentration (MIC). MIC of imipenem and ceftazidime was determined by agar dilution method as recommended by CLSI [15]. Pseudomonas aeruginosa ATCC 27853 was used as a control strain.

Detection of MBL isolates by Ethylenediaminetetraacetic-imipenem test (IPM-EDTA). MBL producing isolates were identified by IPM-EDTA phenotypic method according to the NCCLS recommendation. Briefly, sterilized EDTA 0.5 M (186.1 gr of EDTA in $1000 \mathrm{ml}$ DDW, $\mathrm{pH}$ 8) (pH 8) was prepared. Suspension of the isolates equal to 0.5 McFarland was cultured, and $750 \mu \mathrm{l}$ of $0.5 \mathrm{M}$ EDTA inoculated on imipenem disk $(10 \mu \mathrm{g})$. Imipenem (IMP) and IPM-EDTA disks were placed on the plates. The isolates were considered as MBL producers if the growth inhibition zone around the EDTA-IPM disk was more than the inhibition zone around the IMP disk [16].

Data Analysis. Statistical analysis were performed using SPSS software version 19.0 for Windows (IBM, Chicago, USA). Chi-square and Fisher's exact tests were used to compare proportions. Furthermore, Pairwise correlations were assessed using Phi coefficients. The $P$ values less than 0.05 were considered to be statistically significant.

\section{RESULTS}

Participants' demographic characteristics. Among the $473 \mathrm{P}$. aeruginosa isolates from three hospitals, 58.8\%, and $41.2 \%$ belonged to males and females, respectively. The majority of the isolates were obtained from urine (45.7\%), and the minority from the CSF (5.5\%) (Table 1). The patients' age ranged from 1 to 60 years old, most within the range of 41-60 years old (mean: 58.55), and a few (10\%) were under 10 years old.

Antibiotic susceptibility patterns. The Antibiotic susceptibility pattern of $P$. aeruginosa isolates to different antibiotics is shown in Table 2. The highest resistance rate was observed to ceftizoxime and carbenicillin, and the lowest rate to imipenem and meropenem antibiotics (Table 2).

MIC results. The results of MIC for imipenem and ceftazidime are reflected in Table 3. The MIC of imipenem for all the isolates was between 4 to $64 \mu \mathrm{g} / \mathrm{ml}$, and the majority of the isolates had a MIC $=4 \mu \mathrm{g} / \mathrm{ml}$. Whereas the MIC of ceftazidime for the isolates was between 2 to $>128$ $\mu \mathrm{g} / \mathrm{ml}$, and the majority of the isolates showed a MIC $>128$ $\mu \mathrm{g} / \mathrm{ml}$.

Table 1. The prevalence of MBL-producing $P$. aeruginosa isolated from various samples

\begin{tabular}{l|ll}
\hline Specimen type (n= 665) & No. (\%) of P. aeruginosa isolates (n= 473) & No. (\%) of MBL positive P. aeruginosa isolates (n= 289) \\
\hline Urine & $216(45.7)$ & $142(49.1)$ \\
Sputum & $38(8)$ & $17(5.9)$ \\
Wound & $125(26.4)$ & $69(23.9)$ \\
Blood & $68(14.4)$ & $45(15.6)$ \\
CSF & $26(5.5)$ & $16(5.5)$ \\
\hline
\end{tabular}

Table 2. Antibiotic susceptibility pattern of $P$. aeruginosa isolates

\begin{tabular}{|c|c|c|c|c|c|c|}
\hline \multirow{3}{*}{ Antibiotics } & \multicolumn{6}{|c|}{ Results } \\
\hline & \multicolumn{2}{|c|}{ Sensitive } & \multicolumn{2}{|c|}{ Intermediate } & \multicolumn{2}{|c|}{ Resistant } \\
\hline & Number & Percentage & Number & Percentage & Number & Percentage \\
\hline Imipenem (10 $\mu \mathrm{g})$ & 160 & 33.82 & 7 & 1.48 & 306 & 64.7 \\
\hline Meropenem (10 $\mu \mathrm{g})$ & 153 & 32.34 & 5 & 1.06 & 315 & 66.6 \\
\hline Ceftazidime (30 $\mathrm{\mu g})$ & 109 & 23.05 & 0 & 0 & 364 & 76.95 \\
\hline Carbenicillin $(100 \mu \mathrm{g})$ & 100 & 21.14 & 7 & 1.48 & 366 & 77.38 \\
\hline 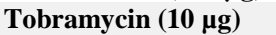 & 118 & 24.95 & 5 & 1.05 & 350 & 74 \\
\hline Amikacin $(30 \mu g)$ & 123 & 26 & 14 & 2.96 & 336 & 71.04 \\
\hline Ceftizoxime ( $30 \mu \mathrm{g})$ & 86 & 18.19 & 0 & 0 & 387 & 81.81 \\
\hline Gentamicin $(10 \mu \mathrm{g})$ & 108 & 22.83 & 6 & 1.27 & 359 & 75.9 \\
\hline
\end{tabular}


Table 3. The results of MIC for imipenem and ceftazidime against 473 clinical isolates of $P$. aeruginosa

\begin{tabular}{|c|c|c|c|c|c|c|c|c|c|c|}
\hline \multirow{2}{*}{ Antibiotics } & \multicolumn{10}{|c|}{ MIC $(\mu \mathrm{g} / \mathrm{ml})$} \\
\hline & 0.5 & 1 & 2 & 4 & 8 & 16 & 32 & 64 & 128 & $>128$ \\
\hline Imipenem (No.) & 0 & 0 & 0 & $352^{*}$ & 72 & 25 & 14 & 10 & 0 & 0 \\
\hline Ceftazidime (No.) & 0 & 0 & $28 *$ & $35^{*}$ & $51^{*}$ & 17 & 21 & 43 & 30 & 248 \\
\hline
\end{tabular}

*Number of susceptible isolates at MIC breakpoints

Detection of MBLs. According to the results of disk diffusion, 306 (64.7\%) isolates were resistant to imipenem, and the phenotype results of MBLs producing $P$. aeruginosa isolates by using IMP-EDTA disk method indicated that 289 (94.5\%) imipenem-resistant isolates were MBL producer. The distribution of the MBL producing $P$. aeruginosa isolates among different clinical samples is reflected in Table 1.

\section{DISCUSSION}

The Pseudomonas-associated infections are as a significant cause of morbidity and mortality in hospitalized patients [13, 17]. Carbapenems such as imipenem and meropenem are the essential class of beta-lactam antibiotics which are used for the treatment of infections caused by the bacteria capable of hydrolyzing penicillin and cephalosporins. Now, MBLs are one of the most important enzymes involved in resistance to carbapenem antibiotics [7]. MBLs producing strains are responsible for chronic and long-term hospital infections [18]. Mortality in severe infections with MBL producing $P$. aeruginosa range from $70 \%$ to $95 \%$ [19]. In a case-control study in Japan, morbidity and mortality among the patients infected with MBLs producing $P$. aeruginosa were higher than those infected by non-MBLs producing isolates [18]. Consequently, the increasing rate of resistance to carbapenems among the bacteria mainly $P$. aeruginosa should be considered as a substantial threat in the treatment of these infections. Furthermore, the rapid spread of MBL genes among pathogens mainly $P$. aeruginosa is considered as the primary concern worldwide that leads to a limitation on the treatment of bacterial infections in different regions of the world $[13,17]$.

In our study, the resistance rate of $P$. aeruginosa isolates to imipenem was $64.7 \%$ using the disk diffusion method. Whereas, previously in similar studies in Iran, the resistance to this antibiotic among the $P$. aeruginosa isolates from hospitalized patients was $32.9 \%$ and $41 \%$, respectively [20, 21]. This result suggests that the imipenem resistance has increased among the $P$. aeruginosa isolates in Iran and it could be an alert for the healthcare system of the country.

Our results also indicated that 289 (94.5\%) isolates of 306 imipenem-resistant isolates produced MBLs by IPMEDTA test. Previously, Van Der Bij and colleagues [22] showed the high accuracy, reproducibility, sensitivity, and specificity of the phenotypic method. In a study by Mihani et al. [23] on 100 P. aeruginosa isolates from burn wards of hospitals of Iran, $42 \%$ isolates showed resistance to imipenem, and 8 (19\%) were MBLs producer. In another report by Sadeghi et al. [24] in Iran on $108 P$. aeruginosa isolates, 40 (37\%) were resistant to imipenem, and 20 (50\%) produced MBLs using IMP-EDTA disk method. In another study in Mashhad, Iran, out of 63 (48.5\%) imipenemresistant isolates, 56 (88.8\%) were MBL-producing [25]. These data suggest that the rate of carbapenem resistance linked to MBLs is increasing in Iran. The high prevalence of MBL producing $P$. aeruginosa may be due to the improper administration of carbapenems. There are also reports of the MBL-producing $P$. aeruginosa isolates from other geographic areas of the world. For instance, the high prevalence of MBL-positive $P$. aeruginosa isolates was reported from Brazil, Korea, Italy, Greece [26-28] and Bangladesh [14]. These results show differences in the prevalence of MBLs producing $P$. aeruginosa isolates in different regions of the world. These differences could be due to the application of different methods for detection of MBLs producing strains, type of Pseudomonas infection, and geographical regions the isolates were collected, and type of disks used.

Our isolates also showed high resistance to other groups of antibiotics including aminoglycosides, cephalosporins, and penicillins which is a characteristic of the majority of MBLs producing isolates. These findings indicate that the usage of these agents for the treatment of $P$. aeruginosa infections in Iran should be limited because of a high resistance rate to these agents among the clinical isolates.

In conclusion, the high prevalence of MBL producing $P$. aeruginosa is alarming. Since the MBLs producing isolates can become resistant to all the beta-lactam antibiotics, detection of carbapenem-resistant $P$. aeruginosa capable of MBLs production can help physicians to choose proper antibiotics for the treatment of the patients infected with these bacteria. The genetic analysis and typing of the MBL enzymes can elucidate the epidemiology of the MBLs producing $P$. aeruginosa isolates.

\section{ACKNOWLEDGEMENT}

The authors would like to thank the staff of Departmant of Molecular Biology, Pasteur Institute of Iran for their crucial technical assistance in this study.

\section{CONFLICT OF INTEREST}

The authors declare that there are no conflicts of interest associated with this manuscript.

\section{REFERENCES}

1. Arvanitidou M, Katikaridou E, Douboyas J, Tsakris A. Prognostic factors for nosocomial bacteraemia outcome: a prospective study in a Greek teaching hospital. J Hosp Infect. 2005; 61 (3): 219-24. 
2. Wolfgang MC, Kulasekara BR, Liang X, Boyd D, Wu K, Yang Q, et al. Conservation of genome content and virulence determinants among clinical and environmental isolates of Pseudomonas aeruginosa. Proc Natl Acad Sci USA. 2003; 100 (14): 8484-9.

3. Bielecki P, Glik J, Kawecki M, dos Santos VAM. Towards understanding Pseudomonas aeruginosa burn wound infections by profiling gene expression. Biotechnol lett. 2008; 30 (5): $777-$ 90.

4. Aguilar-Rodea P, Zúñiga G, Rodríguez-Espino BA, Cervantes ALO, Arroyo AEG, Moreno-Espinosa S, et al. Identification of extensive drug resistant Pseudomonas aeruginosa strains: New clone ST1725 and high-risk clone ST233. PLoS One. 2017; 12 (3): 1-16.

5. Lari AR, Honar HB, Alaghehbandan R. Pseudomonas infections in Tohid Burn Center, Iran. Burns. 1998; 24 (7): 63741.

6. Laupland KB, Parkins MD, Church DL, Gregson DB, Louie TJ, Conly JM, et al. Population-Based Epidemiological Study of Infections Caused by Carbapenem-Resistant Pseudomonas aeruginosa in the Calgary Health Region: Importance of Metallo- $\beta$-Lactamase (MBL)-Producing Strains. J Infect Dis. 2005; 192 (9): 1606-12.

7. Huang Y-T, Chang S-C, Lauderdale T-L, Yang AJ, Wang J-T. Molecular epidemiology of carbapenem-resistant Pseudomonas aeruginosa carrying metallo- $\beta$-lactamase genes in Taiwan. Diagn Microbiol Infect Dis. 2007; 59 (2): 211-6.

8. Senda K, Arakawa Y, Ichiyama S, Nakashima K, Ito H, Ohsuka S, et al. PCR detection of metallo-beta-lactamase gene (blaIMP) in gram-negative rods resistant to broad-spectrum beta-lactams. J Clin Microbiol. 1996; 34 (12): 2909-13.

9. Henrichfreise B, Irith W, Kimberley JS, Bernd W. Detection of VIM-2 metallo-beta-lactamase in Pseudomonas aeruginosa from Germany. Antimicrob Agents Chemother. 2005; 49 (4): 1668-9.

10. Mendiratta D, Deotale V, Narang P. Metallo-beta-lactamase producing Pseudomonas aeruginosa in a hospital from a rural area. Indian J Med Res. 2005; 121: 701-3.

11. Pitout JD, Gregson DB, Poirel L, McClure J-A, Le P, Church DL. Detection of Pseudomonas aeruginosa producing metallo- $\beta$-lactamases in a large centralized laboratory. J Clin Microbiol. 2005; 43 (7): 3129-35.

12. Queenan A, Bush K. Carbapenemases: the Versatile $\beta$ Lactamases. Clin Microbiol Rev. 2007; 20 (3): 440-58.

13. Sacha P, Wieczorek P, Hauschild T, Zórawski M, Olszańska D, Tryniszewska E. Metallo-beta-lactamases of Pseudomonas aeruginosa--a novel mechanism resistance to beta-lactam antibiotics. Folia Histochem Cytobiol. 2008; 46 (2): 137-42.

14. Nasrin T, Jilani MSA, Barai L, Haq JA. Metallo-ßLactamase producing Pseudomonas species in a tertiary care hospital of Dhaka city. Bangladesh J Med Microbiol. 2010; 4 (1): 43-5.

15. CLSI. Performance Standards for Antimicrobial Susceptibility Testing; 18th Informational Supplement. Wayne, PA: Clinical and Laboratory Standards Institute; 2008.
16. Yong D, Lee K, Yum JH, Shin HB, Rossolini GM, Chong Y. Imipenem-EDTA disk method for differentiation of metallo- $\beta$ lactamase-producing clinical isolates of Pseudomonas spp. and Acinetobacter spp. J Clin Microbiol. 2002; 40 (10): 3798-801.

17. Ullah F, Malik SA, Ahmed J. Antimicrobial susceptibility and ESBL prevalence in Pseudomonas aeruginosa isolated from burn patients in the North West of Pakistan. Burns. 2009; 35 (7): 1020-5.

18. Hirakata Y, Yamaguchi T, Nakano M, Izumikawa K, Mine M, Aoki S, et al. Clinical and Bacteriological Characteristics of IMP-Type Metallo-b-Lactamase-Producing Pseudomonas aeruginosa. Clin infect Dis. 2003; 37 (1): 26-32.

19. Cornaglia G, Giamarellou H, Rossolini GM. Metallo-betalactamases: a last frontier for beta-lactams? Lancet Infect Dis. 2011; 11 (5): 381-93.

20. Japoni A, Alborzi A, Kalani M, Nasiri J, Hayati M, Farshad S. Susceptibility patterns and cross-resistance of antibiotics against Pseudomonas aeruginosa isolated from burn patients in the South of Iran. Burns. 2006; 32 (3): 343-7.

21. Khosravi AD, Mihani F. Detection of metallo- $\beta$-lactamaseproducing Pseudomonas aeruginosa strains isolated from burn patients in Ahwaz, Iran. Diagn Microbiol Infect Dis. 2008; 60 (1): 125-8.

22. van der Bij AK, Mol M, van Westreenen M, Goessens WH, Pitout JD. The laboratory diagnosis of Pseudomonas aeruginosa that produce metallo-beta-lactamases in a Dutch tertiary care centre. Scand J Infect Dis. 2011; 43 (8): 596-602.

23. Mihani F, Khosravi A. Isolation of Pseudomonas aeruginosa strains producing metallo beta lactamases from infections in burned patients and identification of blaIMP and blaVIM genes by PCR. Iran J Medical Microbiol. 2007; 1 (1): 23-31.

24. Sadeghi A, Rahimi B, Shojapour M. Molecular detection of metallo- $\beta$-lactamase genes blaVIM-1, blaVIM-2, blaIMP-1, blaIMP-2 and blaSPM-1 in Pseudomonas aeruginosa isolated from hospitalized patients in Markazi province by Duplex-PCR. Afr J Microbiol Res. 2012; 6 (12): 2965-9.

25. Mirbagheri SZ, Meshkat Z, Naderinasab M, Rostami S, Nabavinia MS, Rahmati M. Study on imipenem resistance and prevalence of blaVIM1 and blaVIM2 metallo-beta lactamases among clinical isolates of from Mashhad, Northeast of Iran. Iran J Microbiol. 2015; 7 (2): 72-8.

26. Oh EJ, Lee S, Park YJ, Park JJ, Park K, Kim SI, et al. Prevalence of metallo-beta-lactamase among Pseudomonas aeruginosa and Acinetobacter baumannii in a Korean university hospital and comparison of screening methods for detecting metallo-beta-lactamase. J Microbiol Methods. 2003; 54 (3): 4118.

27. Mansour W, Poirel L, Bettaieb D, Bouallegue O, Boujaafar N, Nordmann P. Metallo-beta-lactamase-producing Pseudomonas aeruginosa isolates in Tunisia. Diagn Microbiol Infect Dis. 2009; 64 (4): 458-61.

28. Irfan S, Zafar A, Guhar D, Ahsan T, Hasan R. Metallo-betalactamase-producing clinical isolates of Acinetobacter species and Pseudomonas aeruginosa from intensive care unit patients of a tertiary care hospital. Indian J Med Microbiol. 2008; 26 (3): 243-5. 